\title{
ENFRENTAMENTO DE FAMILIARES DE PACIENTES EM TRATAMENTO ONCOLÓGICO
}

\section{COPING OF FAMILY OF PATIENTS IN ONCOLOGICAL TREATMENT}

\section{ENFRENTAMIENTO DE FAMILIARES DE PACIENTES EN TRATAMIENTO ONCOLÓGICO}

Fábio da Costa Carbogim ${ }^{1}$, Ramony Zanotti de Assis Pereira ${ }^{2}$, Franciane Silva Luiz ${ }^{3}$, Rodrigo de Oliveira Andrade ${ }^{4}$, Ana Carolina Carraro Tony ${ }^{5}$, Andyara do Carmo Pinto Coelho Paiva ${ }^{6}$

\section{RESUMO}

Objetivo: compreender como os familiares de pessoas em tratamento oncológico lidam com o enfrentamento da doença. Método: estudo descritivo, exploratório de natureza qualitativa, realizado na clínica médica e clínica cirúrgica de um Hospital Oncológico Filantrópico do interior do Estado de Minas Gerais. Foram entrevistados dez acompanhantes de pacientes internados, por meio de um questionário semiestruturado. A seleção foi através de convite aleatório no hospital. Os dados foram analisados utilizando-se a técnica de análise de conteúdo. Resultado: foi identificado mudança de rotina familiar, além do relato de sentimentos de desespero, inconformismo, insegurança, despreparo, angústia e medo de perda do ente acometido pelo câncer. A complexidade e a morosidade dos recursos convencionais intensificam o sofrimento dos familiares. Em contrapartida, a espiritualidade emerge como recurso para enfrentamento da doença. Conclusão: os familiares percebem a espiritualidade/religiosidade como recurso para enfrentar a doença.

Descritores: Enfermagem; Neoplasias; Relações familiares; Cuidadores; Instituições oncológicas.

\footnotetext{
ABSTRACT

Objective: to understand how the relatives of people undergoing cancer treatment deal with the disease. Method: it is a descriptive-exploratory study with a qualitative approach, performed at the medical and surgical clinic of a Philanthropic Oncology Hospital of the State of Minas Gerais, Brazil. Ten companions of hospitalized patients were interviewed through a semistructured questionnaire. The selection was carried out using random invitation at the hospital. Data were analyzed using the content analysis technique. Result: a change in family routine was identified, as well as the report of feelings of despair, lack of acceptance, insecurity, lack of preparation, anguish and fear of losing the loved one affected by cancer. The complexity and

${ }^{1}$ Enfermeiro. Doutor em Ciências. Professor Adjunto da Faculdade de Enfermagem da Universidade Federal de Juiz de Fora

${ }^{2}$ Enfermeira. Residente de Enfermagem em Saúde Mental da Universidade Federal de Juiz de Fora.

${ }^{3}$ Enfermeira. Mestranda em Enfermagem pelo Programa de Pós-Graduação em Enfermagem da Universidade Federal de Juiz de Fora.

${ }^{4}$ Enfermeiro. Mestrando em Enfermagem pelo Programa de Pós-Graduação em Enfermagem da Universidade Federal de Juiz de Fora.

${ }^{5}$ Graduanda em Enfermagem pela Universidade Federal de Juiz de Fora.

${ }^{6}$ Enfermeira. Doutora em Enfermagem. Professora Adjunta da Faculdade de Enfermagem da Universidade Federal de Juiz de Fora.

slowness of conventional resources intensifies the suffering of family members. In contrast, spirituality emerges as a resource for coping with the disease. Conclusion: family members perceive spirituality/religiosity as a resource for coping with the disease.
} 
Descriptors: Nursing; Neoplasms; Family relationships; Caregivers; Cancer Care Facilities.

\section{RESUMEN}

Objetivo: comprender cómo los familiares de personas en tratamiento oncológico tratan con el enfrentamiento de la enfermedad. Método: estudio descriptivo, exploratorio de naturaleza cualitativa, realizado en la clínica médica y clínica quirúrgica de un Hospital Oncológico Filantrópico del interior del Estado de Minas Gerais. Se entrevistó a 10 acompañantes de pacientes internados, a través de un cuestionario semiestructurado. La selección fue a través de invitación al azar en el hospital. Los datos se analizaron utilizando la técnica de análisis de contenido. Resultado: se identificó un cambio de rutina familiar, además del relato de sentimientos de desesperación, no aceptación, inseguridad, despreparo, angustia y miedo a la pérdida del ente acometido por el cáncer. La complejidad y la morosidad de los recursos convencionales intensifican el sufrimiento de los familiares. En cambio, la espiritualidad emerge como recurso para el enfrentamiento de la enfermedad. Conclusión: los familiares perciben la espiritualidad / religiosidad como recurso para enfrentar la enfermedad.

Descriptores: Enfermería; Neoplasias; Relaciones familiares; Cuidadores; Instituciones Oncológicas.

\section{INTRODUÇÃO}

O progressivo envelhecimento da população brasileira tem determinado mudanças em seu perfil de morbimortalidade, o que implica a necessidade de readequações assistenciais, com importante destaque para as doenças não transmissíveis. ${ }^{1}$ Entre as doenças e agravos não transmissíveis, o câncer se destaca e ganha relevância por sua letalidade.

Para o biênio 2018-2019, há uma estimativa de 1,2 milhão de novos casos, sendo os mais incidentes os cânceres de próstata, pulmão, mama feminina, cólon e reto, além dos cânceres de colo uterino, de estômago e de esôfago. ${ }^{1}$

O diagnóstico precoce do câncer e o estabelecimento terapêutico apropriado são importantes fatores de desfecho. ${ }^{2}$ Entre o aparecimento dos primeiros sinais e sintomas e a identificação do câncer, há um percurso complexo nos serviços de saúde que geralmente é percorrido por paciente e familiares. ${ }^{3}$

No tocante às redes de apoio social ao paciente com câncer, a família tem destaque pela proximidade afetiva com o doente, vivenciando todas as incertezas, expectativas, esperanças e necessidades de reorganização cotidiana. ${ }^{3}$ Estudos destacam o apoio familiar oportuno como fator interveniente no acesso e na adesão ao tratamento e um dos principais preditores de manutenção da qualidade de vida e resiliência durante $\mathrm{o}$ tratamento oncológico. ${ }^{4-5}$

Por outro lado, nem sempre os familiares estão preparados para lidar de forma eficaz com o diagnóstico, o tratamento e as orientações terapêuticas, o que pode contribuir para o estresse e 
implicações negativas na qualidade de vida dessas pessoas. ${ }^{5-6}$ Não raro, é possível encontrar familiares e/ou cuidadores com percepções estigmatizadas sobre o câncer, associando-o com morte, finitude, o que resulta em, desde a não menção do nome da doença à omissão do diagnóstico ao paciente. $^{6}$

Nesse contexto, além das intervenções de cunho técnico, cabe aos enfermeiros e demais profissionais de saúde compreender a dinâmica familiar do paciente, valer-se de habilidades de comunicação interpessoal e planejamento como estratégias para minimizar os impactos da doença. ${ }^{3,6}$

Contudo, ainda tem sido premente compreender as vivências de familiares de pacientes em tratamento oncológico como dispositivo para o planejamento assistencial e oferta de serviços adequados. Partindo da premissa de que os familiares de pacientes em tratamento oncológico também sofrem com o adoecimento, emergem indagações sobre as perspectivas, as possibilidades ou desesperanças no processo de cuidado de um ente querido com câncer.

Estudos apontam alta prevalência de sintomas depressivos em familiares de pacientes oncológicos, além de fatores de risco para tristeza, insônia, estresse, alergias e dores no corpo. ${ }^{4-6}$ Essa problemática suscita a realização de estudos que auxiliem e apontem estratégias eficazes no auxílio aos familiares para lidar com a doença. Dessa forma, justifica-se o presente estudo, que tem por objetivo compreender como os familiares de pacientes em tratamento oncológico lidam com o enfrentamento da doença.

\section{MÉTODO}

Trata-se de um estudo descritivoexploratório de natureza qualitativa, realizado na clínica médica e clínica cirúrgica de um Hospital Oncológico Filantrópico do interior do Estado de Minas Gerais.

Participaram do estudo dez familiares, homens e mulheres, acompanhantes de pacientes internados, contatados através de convite aleatório no hospital. Não houve nenhuma recusa ou desistência em participar da pesquisa. Estabeleceu-se como critério de inclusão: familiar maior de 18 anos e que estivesse envolvido diretamente no cuidado ao paciente desde o início da doença. Foram excluídos familiares de pacientes em cuidados oncológicos ambulatoriais.

Os participantes foram esclarecidos sobre os objetivos da investigação e realização da entrevista, consolidando sua anuência através da leitura e assinatura do Termo de Consentimento Livre e Esclarecido. Para assegurar o anonimato e o sigilo das informações prestadas pelos familiares, utilizou-se a letra "P", 
significando participante, seguida da numeração de 1 a 10, de acordo com a ordem de realização das entrevistas.

A coleta dos dados, realizada pelos pesquisadores, ocorreu entre os meses de agosto a setembro de 2016, a partir de um roteiro semiestruturado, contendo dados sociodemográficos como idade, sexo, religião, além das questões norteadoras: como foi que descobriram o câncer em seu familiar? Qual a sua reação e sentimentos com a descoberta do câncer? Quais as necessidades que surgiram a partir da notícia do câncer? Como você tem vivido e enfrentado essa realidade depois da notícia do câncer?

As entrevistas foram individuais, com duração média de dez minutos, gravadas em áudio via gravador digital e posteriormente transcritas na íntegra. A coleta de dados foi realizada até o momento em que foi percebida a repetição frequente do conteúdo manifesto nas falas.

Os dados foram analisados através da técnica de análise de conteúdo ${ }^{7}$, dividida nas seguintes fases: pré-análise, exploração do material e tratamento dos resultados, inferência e interpretação. A pré-análise visa operacionalizar e sistematizar as ideias preliminares, aproximando-se genericamente do texto para compor um corpus de análise. A exploração do material e o tratamento dos resultados reuniram características do corpus por similaridade para, em seguida, por meio de regras de contagem, agregar, classificar e categorizar. $\mathrm{Na}$ fase de inferência e interpretação, procedeu-se à síntese e à estratificação das informações para análise e interpretação crítica dos achados, conduzidos pelos objetivos do estudo.

Cabe destacar que a pesquisa teve início após aprovação do projeto pelo Comitê de Ética em Pesquisa, sob o número de Parecer 1.701.001, CAAE 58364716.1.0000.5147, seguindo-se as recomendações da Resolução n. 466/2012 do Conselho Nacional de Saúde.

\section{RESULTADOS}

Dos dez participantes da pesquisa, a maioria era do sexo feminino, cônjuge do paciente, religião evangélica e com idade média de 46 anos. A identificação e a classificação das unidades de análise das entrevistas fizeram emergir três categorias, apresentadas a seguir:

\section{O descobrimento da doença e sentimentos dos familiares}

Nos relatos abaixo, fica explícito que o descobrimento do câncer causa significativo impacto na vida do familiar. São relatados sentimentos de desespero, inconformismo, despreparo e angústia. No mais, a ausência inicial de informações adequadas desperta o medo da perda, como relatado pelos participantes: 
Um desespero, né? Porque nenhum filho, nenhum familiar está preparado e quer ver isso para uma mãe, né? É de imediato um grande desespero que invade a gente. (P03) Quando soubemos, reunimos no quintal eu e minhas filhas. Elas choravam muito, muito mesmo e eu não tive reação nenhuma. Não aceitava, não sabia o que fazer, mas entreguei tudo na mão do Senhor. (P05)

No começo, é difícil, né? Você fica com um medo. Sem informação de como as coisas vão seguir. (P07)

A gente ficou triste, porque meu pai também passou pelo mesmo problema e faleceu. Aí a gente fica preocupado, sem muita informação, com medo de acontecer a mesma coisa. (P08)

Além disso, após a descoberta do câncer, são comuns sentimentos de inconformismo, de medo da perda, insegurança, causando instabilidade emocional, como verificado nas falas:

Sentimento de dor. É triste, é pior que eu imaginava e, ao mesmo tempo, tenta não passar essa negatividade da doença para ela. (P02)

A gente fica mais é com medo de perder ela, vendo a situação, a gente fica achando que pode acontecer o pior. Na minha família nunca teve nada disso, sabe? Tem sido um baque desde que fiquei sabendo. (P06)

A princípio, falta o chão. Nossa! Depois, foi uma depressão profunda, uma coisa muito triste, abalou a família toda, tanto a minha quanto a família dele. Ficou todo mundo desestabilizado. (P01)

\section{Mudanças no cotidiano familiar}

A família tem sua rotina diária modificada por conta das necessidades de suporte e acompanhamento ao paciente, requerendo ajuda e presença física. Demandas financeiras extras e privações não são incomuns, como pode ser percebido nas falas dos participantes:

Estou faltando muito ao colégio, porque estou olhando ele três vezes por semana. A minha tia teve que vir de Ubá, teve que deixar os filhos dela lá para ajudar. Mudou muito na nossa rotina, ela teve que largaro emprego para poder vir. (P01)

Eu tinha que tomar conta da casa $e$ trabalhar, estava acumulando tudo, tinha que estudar à noite. Ficou muito difícil para mim, tive que deixar as coisas para cuidar dele. Fica difícil fazer outras coisas. (P04) Muda tudo, você para sua vida para viver em função do outro, mas isso, para mim, não é um peso não. Não temos plano de saúde e nós somos de Petrópolis, então aumentou muito os gastos. (P09)

Bastante gastos, mesmo o tratamento sendo pelo SUS, há gastos com combustível, porque moro longe, gasto com alimentação. A minha rotina também mudou, porque eu não saio mais. (P10)

\section{Atitudes diante da doença: recursos convencionais e a espiritualidade}


$\mathrm{O}$ acesso aos recursos convencionais de assistência à saúde, do diagnóstico ao tratamento, é descrito como complexo, moroso e penoso, o que amplia o sofrimento do paciente e da família:

Não é rápido, sabe? Não vai direto ao ponto. Tive que correr muito contra o tempo, pois ficamos quase dois meses indo na UPA. Tratava a dor, fazia medicação e liberava. Até que um dia internaram para oncologia e começou o tratamento. (P06)

Penamos muito, tá? Não sei se você sabe, mas chegar até aqui e começar o tratamento é demorado. Não é isso que falam por aí, que tem prioridade. A cada consulta, achávamos que ia resolver, mas não resolvia. (P03)

Por outro lado, a dimensão espiritual foi reconhecida pelos familiares como um dos principais recursos para o enfrentamento das incertezas, para aceitação e para superação da doença. A espiritualidade, por meio de oração, ritos e interação com um ser superior, é entendida como coadjuvante ao tratamento convencional:

A gente sempre tem a perseverança, né? Pedir a Deus para mostrar o caminho, a solução. Eu sou católico, minha família é católica e minha esperança é em Deus e nos médicos, que vão guiando aqui. (P02)

É só Deus. O que ajuda é a fé, então eu rezo o terço, passo o terço nele, fico lá benzendo ele, essas coisas minha. Sei que primeiro é
Deus, segundo são os tratamentos $e$ profissionais. (P01)

Tenho muita fé em Deus, mas, ao mesmo tempo, tenho medo, não é fácil. As pessoas estão aqui e, de repente, vão. Tenho fé, muita fé em Deus e peço que tire ela dessa situação, desse hospital e do sofrimento. (P03)

\section{DISCUSSÃO}

Os familiares cuidadores de pacientes em tratamento oncológico são, geralmente, parceiros/cônjuges ou parentes em primeiro grau, ascendentes e descendentes, como pais e filhos. Contudo, nem sempre o grau de parentesco é determinante para a prestação de cuidados, uma vez que são encontrados primos, tios, avôs, amigos e até profissionais/cuidadores pagos pela família.

Corroborando outros trabalhos ${ }^{8-10}$, nesta investigação, a maioria dos familiares cuidadores eram mulheres, companheiras/cônjuges dos pacientes. Apesar de importantes mudanças na contemporaneidade em relação às questões de gênero e de provisão de cuidado, ainda é frequente a figura feminina incumbindo-se da assistência e cuidado. ${ }^{9}$

$$
\text { Estudos }^{9-11} \text { apontam não ser }
$$
incomum as companheiras/esposas colocarem como prioridade o cuidado ao companheiro/marido, em detrimento do seu próprio bem-estar. Muitas mulheres perdem ou abandonam o emprego e sofrem alto grau 
de estresse físico e emocional, requerendo, assim como o paciente, assistência à saúde.

O diagnóstico de câncer, termo genérico para doenças crônicas com crescimento desordenado de células e invasão de tecidos, é, no senso comum, frequentemente, associado à morte. ${ }^{12}$ Dessa forma, ao serem noticiados, os familiares e o paciente sofrem grande impacto emocional, o que provoca medo, ansiedade, depressão, frustração, além de alteração na rotina familiar. ${ }^{8,11}$

A forma como a doença é noticiada pode contribuir para desencadear e/ou intensificar o estresse familiar. ${ }^{12}$ Em geral, antes do diagnóstico, o paciente e sua família já passaram por variados serviços de saúde e profissionais e se submeteram a diversos exames, o que os coloca em posição expectante. Essa situação por si só gera angústia e, quando associada a pouca informação, ausência de intervenção multiprofissional e uma comunicação abrupta, muito provavelmente, romperá com equilíbrio e recursos emocionais da família. ${ }^{6,8,10,12}$ Não raro, os familiares e pacientes relatam através de suas experiências que a complexidade e morosidade do sistema de saúde provoca desgaste físico e psíquico.

Com o início do tratamento, novos desafios surgem e repercutem no cotidiano familiar, estabelecendo a necessidade de ajustes, mudanças e abdicações. ${ }^{8}$ Tanto para o paciente quanto para o familiar cuidador, o tratamento e suas consequências interferem na percepção de autonomia e independência dos sujeitos. Isso tem relação com as frequentes internações ou idas a ambulatórios, com comprometimentos funcionais acarretados por efeitos adversos e aumento das demandas financeiras. ${ }^{10,13}$

Sabe-se que a vulnerabilidade social que as doenças oncológicas impõem pode ser intensificada pela precariedade das condições sociais, econômicas e culturais dos pacientes e de seus familiares. ${ }^{14}$ Nesse sentido, estudos ${ }^{13,15}$ indicam que a assistência multiprofissional efetiva, com orientações, planejamento, suporte social, prevenção de agravos evitáveis e intervenções apropriadas, impacta positivamente o tratamento e repercute nos gastos da família. Ensaio clínico ${ }^{16}$ demonstrou que o monitoramento diário dos sinais e sintomas, a partir dos relatos dos pacientes e familiares, influencia significativamente o tratamento e os desfechos clínicos. Além disso, a possibilidade de contato frequente da família com profissionais diminui as sensações de apreensão, insegurança e estresse em um momento caracterizado por sobrecarga.

Se, por um lado, a morosidade para o acesso aos recursos convencionais gera apreensão, a espiritualidade surge como um recurso de enfrentamento e confiança para 
os familiares. A espiritualidade conecta o ser humano ao sagrado e ao transcendente, podendo ou não ser manifesta pela religião. Esta última está vinculada às práticas ritualizadas e aos simbolismos de determinado credo ou cultura. ${ }^{17}$

Destaca-se, pois o papel da espiritualidade/religiosidade enquanto recurso terapêutico em situações de sofrimento psíquico após o diagnóstico de câncer no âmbito familiar. Pesquisas têm demonstrado importante função desse recurso no estímulo a padrões de respostas efetivas ao estresse, ajudando as pessoas a tomarem decisões complexas em um contexto de elevada incerteza. ${ }^{17-19}$

Nesse sentido, tendo em vista o sistema de crenças do paciente e da família, o enfermeiro pode incluir em seu plano de cuidados a espiritualidade/religiosidade como recurso para o fortalecimento do bemestar emocional. Contudo, para seu emprego terapêutico, há necessidade de conhecimento das especificidades das crenças e emprego de ferramentas apropriadas para avaliar as necessidades religiosas e espirituais. ${ }^{18}$

\section{CONCLUSÃO}

A presente investigação permitiu compreender como os familiares de pessoas em tratamento oncológico lidam com o enfrentamento da doença. Foi identificado que, além das mudanças de rotina, os familiares apresentaram sentimentos de desespero, inconformismo, insegurança, despreparo e angústia, bem como o medo de perda do ente com câncer, por falta de informação sobre o tratamento e a doença. Foi evidenciado ainda que o sofrimento do paciente e da família se agrava devido à complexidade e morosidade dos recursos convencionais.

Nesse sentido, a possibilidade de contato dos profissionais de saúde com a família do paciente oncológico, fornecendo informações, esclarecendo dúvidas e valendo-se, além dos recursos convencionais, dos recursos alternativos, como a espiritualidade/religiosidade, pode diminuir as sensações de apreensão, insegurança e estresse.

Como limitação do estudo, destacase que os resultados não podem ser interpretados como representativos de uma população ou grupo de familiares, considerando que foi realizado em dois ambientes de uma mesma instituição hospitalar. Nesse sentido, os resultados devem ser interpretados com cautela e novas pesquisas com abordagens qualitativas e quantitativas são indicadas para ampliar as discussões.

Espera-se que o presente estudo possa contribuir para reflexões sobre a assistência à saúde de familiares de pacientes oncológicos a fim de se determinar estratégias que qualifiquem o 
cuidado integral e fortaleçam o bem-estar emocional dessa população.

Fontes de financiamento: não se aplica.

\section{REFERÊNCIAS}

1. Instituto Nacional do Câncer José Alencar Gomes da Silva. Estimativa 2018: incidência de câncer no Brasil [Internet]. Rio de Janeiro: INCA; 2017 [citado em 16 set 2018]. Disponível em: https://www.inca.gov.br/sites/ufu.sti.inca.1 ocal/files//media/document//estimativaincidencia-de-cancer-no-brasil-2018.pdf

2. Croager EJ, Gray V, Pratt IS, Slevin T, Pettigrew S, Holman CD, et al. Find Cancer Early: evaluation of a community education campaign to increase awareness of cancer signs and symptoms in people in regional Western Australians. Front Public Health [Internet]. 2018 [citado em 16 set 2018]. Disponível em:

https://www.ncbi.nlm.nih.gov/pmc/articles /PMC5809399/

3. Lima BC, Silva LF, Góes FGB, Ribeiro MTS, Alves LL. O itinerário terapêutico de famílias de crianças com câncer: dificuldades encontradas neste percurso. Rev Gaúch Enferm. [Internet]. 2018 [citado em 16 set 2018]; 39:e2018-0004. Disponível em: https://seer.ufrgs.br/RevistaGauchadeEnfer magem/article/viewFile/86447/49696

4. Costa ALS, Heitkemper MM, Alencar GP, Damiani LP, Silva RM, Jarrett M E. Social support is a predictor of lower stress and higher quality of life and resilience in brazilian patients with colorectal cancer. Cancer Nurs. [Internet]. 2017 [citado em 16 set 2018]; 40(5):352-60. Disponível em: https://www.ncbi.nlm.nih.gov/pubmed/271 71810

5. Swartzman S, Sani F, Munro A J. The role of social support, family identification, and family constraints in predicting posttraumatic stress after cancer. PsychoOncology. [Internet]. 2017 [citado em 16 set 2018]; 26(9):1330-35. Disponível em: https://www.ncbi.nlm.nih.gov/pubmed/278 62598

6. Piolli KC, Decesaro MN, Sales CA. O (des) cuidar-se como mulher ao ser cuidadora do companheiro com câncer. Rev Gaúch Enferm. [Internet]. 2018 [citado em 16 set 2018]; 39:e2016-0069. Disponível em: https://seer.ufrgs.br/RevistaGauchadeEnfer magem/article/view/79528

7. Bardin L. Análise de conteúdo. São Paulo: Edições 70, 2011.

8. Piolli KC, Medeiros M, Sales CA. Significações de ser cuidadora do companheiro com câncer: um olhar existencial. Rev Bras Enferm. [Internet]. fev 2016 [citado em 16 set 2018]; 69(1):110-17. Disponível em: http://www.scielo.br/pdf/reben/v69n1/0034 -7167-reben-69-01-0110.pdf

9. Borges EL, Franceschini J, Degani Costa LH, Fernandes ALG, Jamnik S, Santoro IL. Family caregiver burden: the burden of caring for lung cancer patients according to the cancer stage and patient quality of life. J Bras Pneumol. [Internet]. 2017 [citado em 16 set 2018]; (43):18-23. Disponível em:

https://www.ncbi.nlm.nih.gov/pubmed/281 25154

10. Wood R, Taylor-Stokes G, Lees M. The humanistic burden associated with caring for patients with advanced non-small cell lung cancer (NSCLC) in three European countries a real world survey of caregivers. Support Care Cancer. [Internet]. 2018 [citado em 16 set 2018]; (26):1-11. Disponível em: https://www.ncbi.nlm.nih.gov/pubmed/301 21787

11. Milagres MAS, Mafra SCT, Silva EP. The impact of cancer on the everyday life of women in their family nucleus. Ciênc Cuid Saúde [Internet]. 2016 [citado em 16 set 2018]; 15(4):738-45. Disponível em: http://www.periodicos.uem.br/ojs/index.ph p/CiencCuidSaude/article/view/29893/184 64

12. Figueiredo T, Silva AP, Silva RMR, Silva JJ, Silva CSO, Alcântara DDF, et al. Como 
posso ajudar? Sentimentos e experiências do familiar cuidador de pacientes oncológicos. ABCS Health Sci. [Internet]. 2017 [citado em 16 set 2018]; 42(1):34-39. Disponível em:

https://www.portalnepas.org.br/abcshs/arti cle/view/947

13. Graham J, Gingerich J, Lambert P, Alamri A, Czaykowski P. Baseline Edmonton Symptom Assessment System and survival in metastatic renal cell carcinoma. Current Oncol. [Internet]. 2018 [citado em 16 set 2018]; 25(4):e319-e323. Disponível em: https://www.ncbi.nlm.nih.gov/pubmed/301 11978

14. Paula DPS, Silva GRC, Andrade JMO, Paraiso AF. Cancer in children and adolescents in the family settings: perceptions and experiences with respect to their diagnosis. Rev Cuid. (Bucaramanga). [Internet]. 2019 [citado em 16 jan 2019]; 10(1):e570. Disponível em: https://revistacuidarte.udes.edu.co/index.ph p/cuidarte/article/view/570/1049.

15. Strasser F, Blum D, von Moos R, Cathomas R, Ribi K, Aebi S, et al. The effect of real-time electronic monitoring of patient-reported symptoms and clinical syndromes in outpatient workflow of medical oncologists: E-MOSAIC, a multicenter cluster-randomized phase III study (SAKK 95/06). Ann Oncol. [Internet]. 2016 [citado em 16 set 2018]; 27(2):324-32. Disponível em: https://www.ncbi.nlm.nih.gov/pubmed/266 46758

16. Basch E, Deal AM, Kris MG, Scher HI, Hudis CA, Sabbatini P, et al. Symptom monitoring with patientreported outcomes during routine cancer treatment: a randomized controlled trial. J Clin Oncol. [Internet]. 2016 [citado em 16 set 2018]; 34(6):557-65. Disponível em: https://www.ncbi.nlm.nih.gov/pubmed/266 44527

17. Nejat N, Whitehead L, Crowe M. The use of spirituality and religiosity in coping with colorectal cancer. Contemp Nurse. [Internet]. 2017 [citado em 16 set 2018]; 53(1):48-59. Disponível em: https://www.ncbi.nlm.nih.gov/pubmed/280 06988

18. Jimenez-Fonseca P, Lorenzo-Seva U, Ferrando PJ, Carmona-Bayonas A, Beato $\mathrm{C}$, García $\mathrm{T}$, et al. The mediating role of spirituality (meaning, peace, faith) between psychological distress and mental adjustment in cancer patients. Support Care Cancer. [Internet]. 2018 [citado em 16 set 2018]; 26(5):1411-18. Disponível em:

https://www.ncbi.nlm.nih.gov/pubmed/291 43135

19. Alvarenga JTA, Molina NPFM, Silva GRD, Silva LMA, Rodrigues RL. Perfil socioeconômico, demográfico e indicativo de depressão em mulheres submetidas à mastectomia no pós-operatório tardio. Rev Enferm Atenção Saúde [Internet]. ago/set 2018 [citado em 24 nov 2018]; 7(2):3-16. Disponível em:

http://seer.uftm.edu.br/revistaeletronica/ind ex.php/enfer/article/view/1846

RECEBIDO: $24 / 11 / 18$

APROVADO: 04/04/19

PUBLICADO: 07/19 